\title{
Intracerebral Pro-inflammatory Cytokine Increase in Surgically Evacuated Intracerebral Hemorrhage - an Observational Microdialysis Study
}

Lovisa Tobieson ( $\square$ lovisa.tobieson@regionostergotland.se )

Linköping University: Linkopings universitet https://orcid.org/0000-0003-2284-846X

Anna Gard

Lunds Universitet

Karsten Ruscher

Lund University: Lunds Universitet

Niklas Marklund

Lund University: Lunds Universitet

\section{Research}

Keywords: cytokine, inflammation, intracerebral hemorrhage, microdialysis, secondary brain injury

Posted Date: May 24th, 2021

DOI: https://doi.org/10.21203/rs.3.rs-516589/v1

License: (c) (i) This work is licensed under a Creative Commons Attribution 4.0 International License. Read Full License 


\section{Abstract}

\section{Background:}

Treatment options for spontaneous intracerebral hemorrhage $(\mathrm{ICH})$ are limited. A possible inflammatory response in the brain tissue surrounding an $\mathrm{ICH}$ may exacerbate the initial injury and could be a target for treatment.

\section{Methods:}

In this observational study, ten patients needing surgical evacuation of supratentorial ICH received two cerebral microdialysis (MD) catheters; one in the perihemorrhagic zone (PHZ), and one in non-eloquent cortex (SNX) remote from the ICH. The microdialysate was analysed for energy metabolites (including lactate/pyruvate ratio (LPR) and glucose) and for inflammatory mediators using a multiplex immunoassay of 27 cytokines and chemokines at 6-10 hours, 20-26 hours and 44-50 hours after surgery.

\section{Results:}

Deranged energy metabolic markers suggestive of a metabolic crisis were found in $\mathrm{PHZ}$ compared to SNX, persistent throughout the 50 hours. Pro-inflammatory cytokines IL-8, TNF-a, IL-2, IL-1 $\beta$, IL-6 and IFN$\gamma$, anti-inflammatory cytokine IL-13, IL-4, and VEGF-A were significantly higher in PHZ compared to SNX, most prominent at 20-26 hours following $\mathrm{ICH}$ evacuation.

\section{Conclusions:}

Higher levels of pro- and anti-inflammatory cytokines in the perihemorrhagic brain tissue suggests a role for inflammatory mediators involved in secondary injury cascades potentially exacerbating tissue injury, which may constitute a target for future medical interventions.

\section{Background}

Spontaneous supratentorial intracerebral hemorrhage $(\mathrm{ICH})$ represents the most severe form of stroke, as only $20 \%$ of patients return to normal activities of daily living following $\mathrm{ICH}(1)$. Current medical treatment options include correction of coagulation disorders and, potentially, acute blood pressure lowering to prevent ICH expansion(2,3), but specific medical therapies to treat $\mathrm{ICH}$ are still lacking. Furthermore, since the role of surgery in improving outcome in larger cohorts of ICH patients remains controversial,(4-6) the treatment options for $\mathrm{ICH}$ remain limited.

The rupture of a blood vessel and the associated ICH causes an initial primary injury to the brain by a direct tissue destruction, and a possible increased intracranial pressure (ICP). Following this primary injury, the blood break down products, including haemoglobin and its metabolites, and/or the increased ICP cause additional secondary brain injury by initiating reactive cellular, metabolic and neurotoxic cascades in the surrounding tissue. The brain tissue surrounding an $\mathrm{ICH}$, the perihemorrhagic zone (PHZ), 
shows an acute hypometabolic and hypoperfusion state(7-9) including mitochondrial dysfunction and metabolic failure, $(10,11)$ and this region may be particularly vulnerable to secondary injury. Furthermore, in experimental ICH studies an inflammatory response has been observed that includes rapid activation of microglia,(12-15) followed by the infiltration of inflammatory cells over hours and days.(16) This inflammatory response may result in breakdown of the blood-brain barrier exacerbating the vasogenic oedema and tissue damage.(16) In animal ICH models, neurologic outcome can be improved by attenuating the inflammatory process.(17-23)

There are no available pharmacological treatment options with proven clinical benefit targeting the secondary injury following $\mathrm{ICH}$.(24) Clinical studies using early glucocorticoid administration in an attempt to attenuate the ICH-induced inflammation resulted in an impaired clinical outcome.(25) These results emphasize the complex contribution of inflammation to post-ICH outcome and since the inflammatory response is necessary as part of the healing process, it is essential to understand the dynamics and complex interaction of inflammatory mediators in brain tissue following ICH.

Cerebral microdialysis (MD) is a useful clinical tool used to monitor the microenvironment of the brain tissue in the neurocritical care patient. MD has been extensively used in human traumatic brain injury (TBI) and subarachnoid hemorrhage (SAH) although only rarely in clinical ICH research.(11, 26-29) In recent years, and with the introduction of catheters with a larger pore size, MD has enabled sampling of inflammatory mediators from the interstitial fluid of brain tissue in humans $(30,31)$ and in animal models. (32) The cytokine expression has been explored in SAH and TBI patients,(31, 33-38) demonstrating cerebral production of cytokines,(36) but no such studies exist in ICH. Furthermore, several studies have explored the concentration of various pro-inflammatory mediators in plasma samples of ICH patients, showing a correlation between levels of IL-6,(39-42) TNF- $a(41,42)$ and poor outcome, supportive of the hypothesis that inflammation plays a role in secondary brain injury following $\mathrm{ICH}$.

In this study, we sampled interstitial fluid using paired MD catheters, one in the perihemorrhagic zone (PHZ) and one in seemingly normal and non-eloquent cortex (SNX), to investigate changes in inflammatory mediators in the acute phase after surgical evacuation of $\mathrm{ICH}$. Following analysis of routine low-molecular weight metabolites (glucose, lactate, pyruvate, glycerol, glutamate), the remaining microdialysate was investigated by a Multiplex immunoassay. We hypothesized a difference in pro- and anti-inflammatory cytokine and chemokine expression in the PHZ compared to SNX, indicative for distinct inflammatory profiles in these brain areas.

\section{Methods}

\section{Patients}

Adult patients $>18$ years old and requiring emergent surgery for $\mathrm{ICH}$ using an open craniotomy, at the Department of Neurosurgery, University Hospital Linköping, Sweden, during the period 2016-2018 were prospectively recruited to the study. Exclusion criteria included severe coagulation disorders and a known source of bleeding, such as an aneurysm or an arteriovenous malformation. The ICH was evacuated via a 
standard craniotomy and by microneurosurgical evacuation. Intracranial pressure (ICP)-monitoring was achieved using either Neurovent-P parenchymal pressure monitoring device (Raumedic AG, Helmbrechts, Germany) or Bactiseal external ventricular drainage (EVD) catheter (DePuy Synthes, Raynham, USA). One microdialysis (MD) catheter (CMA-71 Brain Catheter, M-Dialysis, Solna, Sweden) was inserted via the craniotomy into the perihemorhagic zone (PHZ) aiming for within $1 \mathrm{~cm}$ of the evacuated $\mathrm{ICH}$, and one catheter was inserted ipsilateral to the hemorrhage in seemingly normal and non-eloquent cortex (SNX). MD catheters were inserted at a 45 degree angle aiming for the microdialysis membrane to include the cortex-white matter junction. As it is essential for the interpretation of MD sampling to know the location of the membrane $(43,44)$ a post-operative CT-scan was performed to verify MD catheter placement.

Three of the patients were included in a previous publication from our group evaluating energy metabolic disturbances using MD following ICH surgery.(29)

Patients were treated according to a standardized neurocritical care protocol to avoid secondary insults. This included intubation and mechanical ventilation if the patient was unconscious (Glasgow Coma Scale motor score (GCS-M) $\leq 5$ ), aiming for normoventilation, normovolemia, and normothermia. ICP was maintained at $\leq 20 \mathrm{mmHg}$ and cerebral perfusion pressure (CPP) $>60 \mathrm{mmHg}$ with the use of volume substitution and inotropic drugs such as norepinephrine or dobutamine, when needed.

To determine outcome according to modified Rankin Scale (mRS) patients or their closest relative were asked to complete a validated questionnaire at 3-6 months post ICH-onset.(29, 45, 46)

\section{Microdialysis}

Microdialysis catheters of $10 \mathrm{~mm}$ length with a molecular weight cut-off (MWCO) of $100 \mathrm{kDa}$ (CMA-71, M-dialysis AB, Solna, Sweden) were used. The catheters were perfused per institutional routine with $5 \%$ human albumin in a water solution containing the excipients sodium chloride, $\mathrm{N}$-acetyl-DL-tryptophan and caprylic acid (Albunorm, 50g/l, Octapharma AB, Stockholm, Sweden), at a rate of $0.3 \mu \mathrm{L} / \mathrm{min}$ using the CMA 106 perfusion pump (M-Dialysis AB, Solna, Sweden).(31) The first 2 hours (0-2) of sampling were discarded according to consensus praxis. $(44,47)$ Samples were collected every 2 hours for routine analysis of small molecular metabolites (glucose, lactate, pyruvate, glycerol and glutamate). $(44,47-49)$ Following this analysis, the remaining MD sample (approximately $30 \mu \mathrm{L} / \mathrm{vial}$ ) was frozen and stored at $20^{\circ} \mathrm{C}$, and typically within 2-8 weeks transferred to Eppendorf vials and stored at $-86^{\circ} \mathrm{C}$ until further analysis.

\section{Analytical Methods}

\section{Metabolite analysis}

Interstitial levels of glucose, lactate, pyruvate, glycerol and glutamate in the MD samples were analysed bedside using an ISCUS Flex ${ }^{\circledR}$ analyser (M Dialysis AB, Solna, Sweden). The lower limit of detection (LLOD) was $1.0 \mu \mathrm{mol} / \mathrm{L}$ for glutamate, $0.1 \mathrm{mmol} / \mathrm{L}$ for glucose and lactate, $10 \mu \mathrm{mol} / \mathrm{L}$ for pyruvate and $0.22 \mathrm{mg} / \mathrm{mL}$ for glycerol. No sample preparation was needed. Metabolite concentrations in the MD 
samples were analysed by the enzymatic methods using the ISCUS Flex® analyser, immediately after sample collection. The sample volume required for the different metabolites was $0.5 \mu \mathrm{L}$ for glucose, 0.2 $\mu \mathrm{L}$ for lactate, $0.5 \mu \mathrm{L}$ for pyruvate, $0.5 \mu \mathrm{L}$ for glycerol, $1 \mu \mathrm{L}$ for glutamate and $0.5 \mu \mathrm{L}$ for urea. Following the analysis of these metabolites, approximately $30 \mu \mathrm{L}$ of dialysate remained that were used in the Multiplex immunoanalysis.

\section{Multiplex immunoassay}

MD samples from three different time-periods were analyzed for inflammatory mediators using the MSD (Meso Scale Discovery, Rockville, USA) MULTI-SPOT Assay System V-PLEX Human Proinflammatory Panel 1, Cytokine Panel 1 and Chemokine Panel 1(cat \#K15210D). The proteins in each panel were detected by immunoassays. Antibodies with electrochemiluminescent labels bind the proteins, the MSD instrument applies a voltage to emit light from these labels and measures the intensity of the light, providing a quantitative measure of each protein concentration.

Pooling of three MD vials, after analysis of the routine analytes, was needed to achieve a sufficient sample volume of $25 \mu \mathrm{l}$ per well enabling the analysis and thus a 6-hour time resolution was achieved. The three chosen time points were; early (4-10 hours), intermediate (20-26 hours) and late (44-50 hours after surgery). The pooled samples were diluted to $\otimes$ of the original concentration by adding $11 \mu \mathrm{L}$ sample to $44 \mu \mathrm{L}$ diluent.

All samples were analyzed with the same protocol. Pooling of samples was done $24 \mathrm{~h}$ before the analysis of the plates, pooled samples was stored at $-20^{\circ} \mathrm{C}$ before analysis.

\section{Antibody Solution}

V-PLEX Proinflammatory Panel 1: $67 \mu \mathrm{L}$ of SULFO-TAG Anti-human IFN-y, IL-1 $\beta$, IL-2, IL-4, IL-6, IL-8, IL-10, IL-13 and TNF-a were added to $2400 \mu \mathrm{L}$ of diluent.

V-PLEX Cytokine Panel 1: $67 \mu$ L of SULFO-TAG Anti-human IL-1a, IL-5, IL-7, IL-12/IL-23p40, IL-15, IL-16, IL$17 \mathrm{~A}, \mathrm{TNF}-\beta$ and VEGF-A were added to $2400 \mu \mathrm{L}$ of diluent.

V-PLEX Chemokine Panel 1: $67 \mu \mathrm{L}$ of SULFO-TAG Anti-human Eotaxin, MIP-1 $\beta$, Eotaxin-3, TARC, IP-10, MIP-1a, MCP-1, MDC and MCP-4 were added to $2400 \mu \mathrm{L}$ of diluent.

Assay Protocol

After blocking using the blocker $\mathrm{H}$ for one hour with shaking for one hour, plates were washed three times with $150 \mu \mathrm{L}$ Wash Buffer (PBS $+0.05 \%$ Tween 20). Thereafter, $25 \mu \mathrm{L}$ sample or calibrator were added. The plates were sealed and incubated at room temperature with shaking for two hours. The plates were washed three times with $150 \mu \mathrm{L}$ Wash Buffer, after which $25 \mu \mathrm{L}$ of antibody solution was added to each well. The plates were sealed and incubated at room temperature with shaking for two hours, and 
subsequently washed three times with $150 \mu \mathrm{L}$ Wash Buffer. To each well, $150 \mu \mathrm{L}$ of 2 X Read Buffer T was added, and the plates were analyzed with a MESO QuickPlex SQ 120 instrument.

\section{Statistical analyses}

Statistical analyses were performed in SIMCA 15.0.2 (Umetrics, Sweden) or in IBM SPSS 27.0 (IBM, Kista, Sweden).

Data distribution was assessed using Shapiro-Wilks' normality test. Normally distributed data are presented as mean and standard deviation (SD). Non-normally distributed data are presented as median and range, median and interquartile range or median and individual values.

Low-molecular weight metabolite data were analysed using linear mixed model (MML) method using patient number as subject level and catheter location as fixed effect.

Multivariate analysis (MVA) was performed by overviewing the data using principal component analysis (PCA) and thereafter fitting orthogonal projections to latent structures discriminant analysis (OPLS-DA) model to the data. Critical outliers were investigated with Hotelling's T2, a multivariate generalization of confidence interval, in the PCA. Any data point outside the resulting $>99 \%$ confidence interval was removed from analysis. Moderate outliers were investigated using distance to model X (DModX)(50). Model validity was investigated with a cross-validated ANOVA (CV-ANOVA) and a p-value $<0.05$ considered significant. R2 and Q2 are presented for the OPLS-DA model along with number of latent variables. Scaling to unit variance and mean centering was employed. Variables with a $\mid p($ corr) $\mid>0.4$ and VIP $>1$ were considered significant.(50)

Univariate analysis was performed using paired t-test for normally distributed data and paired Wilcoxon signed rank test for non-normally distributed data.

In the cytokine data analysis, values below lower limit of detection (LLOD) of the assay were substituted with the exact value given for LLOD by the manufacturer.

\section{Results}

Ten patients ( 8 males, 2 females; Table 1) underwent surgery with evacuation of intracerebral hemorrhage (ICH) and placement of dual microdialysis (MD) catheters (Figure $1 a-b)$ and were thus included in the study. Median age was 64 (51-71) years; median time to surgery 27.8 (6-82) hours and median MD sampling time was 95 (50-148) hours. Mean distance from MD catheter to evacuated ICH was $6( \pm 5.0) \mathrm{mm}$ for the $\mathrm{PHZ}$ catheter and $24.5( \pm 7.6) \mathrm{mm}$ for the SNX catheter. Mean volume of ICH was 77 ( \pm 25.8$) \mathrm{mL}$ (Table 1). The PHZ catheter of patient 10 was not placed according to protocol as it was found to be located $>10 \mathrm{~mm}$ from the hematoma cavity. However, since it was on CT control located in tissue affected by the ICH and the surgical approach, it was included in the analysis. The SNX catheter of patient 7 was placed in parenchyma in which an ischemic infarction developed post-surgery, thus an area 
not representing seemingly normal cortex, and the data from this MD catheter was excluded from further analysis.

\section{Metabolites}

MD-Glucose levels were significantly lower in PHZ compared to SNX (Figure 2a; $p<0.05$ ), however, in both locations MD-glucose levels were consistently above the $0.2 \mathrm{mmol} / \mathrm{L}$ critical level,(47) suggesting adequate substrate delivery. Nevertheless, the lactate pyruvate ratio (LPR) was significantly higher in $\mathrm{PHZ}$ compared to SNX (Figure 2b; $p<.05$ ) indicative of a metabolic crisis in the PHZ.(29) Although the LPR elevation in the $\mathrm{PHZ}$ was particularly evident early following surgery, it persisted beyond 48 hours. MDglutamate and MD-glycerol levels were also significantly higher in PHZ compared to SNX (figure 2c-d; $\mathrm{p}<$ $0.05)$.

\section{Cytokines and chemokines}

All cytokines were in detection range in the microdialysate although the expression of each cytokine was highly variable with the highest and lowest levels differing by a 4 -fold dynamic range. TNF- $\beta$ was recovered in less than $2 \%$ of the samples and was therefore excluded from analysis. In total 1512 multiplex analysis results were obtained.

\section{Univariate analysis}

Univariate analysis of cytokines and chemokines revealed a significantly higher level of IL-2, IL-8 and IL$1 a$ at 20-26 hours post-surgery whereas IL-6 and IL-4 levels were increased at 44-50 hours in the PHZ when compared to the SNX (Figure 3; $p<0.05$ ). Several cytokines, including most anti-inflammatory ones, displayed a similar pattern in the PHZ and the SNX (Supplemental figure I). Chemokines generally displayed a pattern of higher levels in the SNX when compared to PHZ, however, only TARC levels were statistically different (Supplemental figure II).

\section{Multivariate analysis}

A multivariate analysis with unsupervised principal component analysis (PCA) illustrated clustering in the data, showing some group separation (Figure 4a). Hotelling's T2 analysis showed two critical outliers (>99\% T2) which were subsequently removed. Neither global nor pair wise tests results were changed by removal of these outliers. Score scatter plot for PCA showed inherent group separation in the data (Figure 4a). The corresponding loading scatter plot showed PHZ data clustering largely due to metabolites and pro-inflammatory cytokines (Figure $4 \mathrm{~b}$ ). The pro-inflammatory and metabolite variables clustered near time point 2 (corresponding to 20-26 hours post-surgery) as opposed to time points 1 and 3 (corresponding to 4-10 and 46-50 hours post-surgery, respectively).

A supervised OPLS-DA model was then fitted (OPLS-DA (1+0+0) R2 0.179, Q2 0.23; p<.001) with MD catheter location as class (Figure 4c). The OPLS-DA model was used to evaluate which metabolites and cytokines discriminate between the two MD catheter locations. To determine which variables contributed 
most strongly to the model $\mid \mathrm{p}$ (corr) $\mid>0.4$ and VIP $>1$ was used, resulting in four metabolites and eight cytokines (Table 2) showing the strongest contribution to the difference between the PHZ and SNX. Notably, low molecular weight metabolites suggestive of a metabolic crisis and tissue injury such as lactate, LPR, pyruvate and glutamate, were significantly higher in PHZ when compared to the SNX. Furthermore, the pro-inflammatory cytokines TNF-a, IL-6, IFN- - , IL-1ß, IL-8, and IL-2 were higher in PHZ compared to SNX, as were the anti-inflammatory cytokines IL-13 and IL-4, and growth factor VEGF-A.

\section{Discussion}

We used an approach of placing paired microdialysis catheters, one in the vicintiy of the intracerbral hemorrhage $(\mathrm{ICH})$ into the perihemorrhagic zone $(\mathrm{PHZ})$ and one into non-injured brain tissue in a region not affected by the surgical approach or the ICH- the seemingly normal cortex (SNX). Our key findings were that both univariate and multivariate analysis showed a higher expression of predominantly proinflammatory cytokines in the PHZ when compared to the SNX area. These differences were most evident at 20-26 hours, although some pro-inflammatory cytokines peaked at the later $44-50$ hours post-surgery interval. Pro-inflammatory cytokines IL-8, TNF-a, IL-2, IL-1 $\beta$, IL-6 and IFN- $\gamma$, were significantly higher in $\mathrm{PHZ}$ compared to SNX. Increased pro-inflammatory cytokine expression in perihemorrhagic tissue was previously observed in animal models including expression of IL-1 $\beta$, TNF-a, and MIP-1a (18, 51-54), although there are no previous studies of cytokine expression in brain tissue of $\mathrm{ICH}$ patients. Our present findings support the presence of a pro-inflammatory environment in the tissue surrounding an ICH and provides information on the temporal profile of the inflammatory cascades. VEGF-A, which mediates increased permeability of the blood-brain-barrier $(\mathrm{BBB})(55,56)$, was distinctly higher in $\mathrm{PHZ}$ compared to SNX, which may thus potentially aggravate vasogenic edema and cause further secondary brain injury.

Analysis of low-molecular weight metabolites showed a metabolic crisis in the perihemorrhagic tissue, persisting beyond the initial 48 hours post-surgery, consistent with a previous study by our group.(29) The initial high level of MD-glutamate in PHZ suggests an ongoing neuronal death immediately following surgery and a subsequent plausible cell membrane degradation could contribute to the increase in MDglycerol seen over time.

There is also a beneficial role for the inflammatory response in $\mathrm{ICH}$, in particular the $\mathrm{M} 2$ phenotype of microglia, involved in clearing debris and repair of tissue following ICH(12-15, 18-20, 23, 57-63). IL-13 and IL-4 are known to be anti-inflammatory or have a modulating effect on other pro-inflammatory mediators(64) and in the present study were increased in the PHZ. IL-4 has been shown to convert M1 phenotype microglia toward an M2 phenotype, $(65,66)$ further illustrating that tissue reaction to ICH is complex and the balance of pro- and anti-inflammatory mediators may shift over time.

Cytokines can have both a dual and conflicting role. To classify them as either anti- or pro-inflammatory may be overly simplistic, as the immune modulators constitue an intricate network of interconnected paracrine and autocrine molecules whith both positive and negative feed-back loops inherent to the system.(67) Therefore, it is important to consider both the context, the timing and the target of any given 
cytokine in order to understand and interpret its action as either a driver or a moderator of the immune response.(68) In particular IL-6, TGF- $\beta$ and IFN- $y$ can be either pro- or anti-inflammatory depending on timing and target.(68) In similarity to our present study, both anti- and proinflammatory cytokines are released simultaneously in the tissue following TBI. $(69,70)$

One known limitation of intracerebral microdialysis is the lack of control levels, and interpretation of absolute levels must be made with caution. Our approach enables evaluation of the temporal profile of the inflammatory cascades but also a comparison to a relatively uninjured brain region. While the SNX is in a region exposed by the surgical approach, it is not injured by the surgery. While we cannot exclude an inflammatory response also in the SNX, our data argue for more marked changes occurring in the PHZ. Furthermore there are known challenges with interpretation of microdialysis data with regards to relative recovery, which is dependent on several factors, $(47,71)$ and which was not determined in the present study. However, relative recovery can be assumed to be similar between the paired catheters, thus differences between these should not be affected by changes in recovery.

Here, we did not measure inflammatory mediators in blood plasma or cerebrospinal fluid. Previous studies have shown elevated serum levels of IL-6 and TNF-a in ICH patients,(39-42) suggesting some mediators of the inflammatory response can be monitored also in blood. Cytokines generally have a paracrine target, however, and as such the cytokine expression in brain tissue can be expected to contribute to ongoing inflammatory response without any measurable corresponding increase in plasma concentration. In addition, a previous study of 22 TBI patients showed the concentration of cytokines to be several times higher in cerebral interstitial fluid, measured by microdialysis, than in blood.(36) The same study showed recovery of 42 cytokines from the interstitial fluid, and demonstrated distinct temporal profiles in 16 of those 42 . Similarly, in the present study, TNF-a, IL-8, IL-1 $\beta$ and IL- 6 were found to peak within the first two days of monitoring,(36) although there was no comparison of peri-contusional and more normal tissue. Of note, while $\mathrm{TBI}$ and $\mathrm{ICH}$ may share some characteristics, they are also two distinct disease entities with unique pathophysiology. Thus, the data in this study are highly novel since cytokine expression in $\mathrm{ICH}$ patients has rarely been measured, and only as a part of a mixed cohort of neurointensive care patients including both SAH and TBI patients. $(33-35,72)$

\section{Limitations}

This study is limited by the small number of patients, explained by the highly complex set-up including the technical challenge of inserting paired microdialysis catheters, which limits the statistical power. To date, only $4-5 \%$ of all ICH patients undergo surgical evacuation(73) which precludes gathering large number of patients.

Due to the unique set-up of this present study we were able to compare the perihemorrhagic expression of cytokines to those in control brain tissue. This control tissue, while not directly targeted by the surgical approach nor influenced by the $\mathrm{ICH}$, it should not be regarded as entirely uninjured. However, the distinct differences between the two catheters argues against against a marked tissue reaction and cytokine release from insertion of the microdialysis catheter per se. Due to ethical reasons the insertion of 
microdialysis catheters in $\mathrm{ICH}$ patients who do not undergo surgical evacuation is not possible, therefore there are no control patients in our cohort without craniotomy. The surgical approach, performed using microsurgical technique to minimize the trauma to the surrounding tissue, may have contributed to the release of inflammatory mediators.

In this present study we do not report on outcome of the tissue surrounding MD catheters. Therefore, we cannot say that the demonstrated increase in pro-inflammatory cytokine expression in $\mathrm{PHZ}$ is detrimental to tissue outcome, however, previous animal studies show improved neurological function and tissue outcome following augmentation of inflammatory responses. $(51,74-76)$

The low number of measured time points (three) limit the time resolution and preclude the discovery of cytokines possibly peaking later than 50 hours as has previously been demonstrated for IL-12p70 and IL10 in TBI patients.(36) It is plausible that some protein expression may have been elevated beyond the studied 50 hours. Nevertheless, our results show that the most significant changes occurred already at 20-26 hours after surgery suggesting an early inflammatory peak following $\mathrm{ICH}$. It is plausible that antiinflammatory cytokines may also have an increasingly important role in the $\mathrm{PHZ}$ over a longer period of time, thus beyond the scope of our study design. Furthermore, we do not know the concentration of cytokines prior to the insertion of the MD catheters, thus any peak prior to MD insertion would also evade detection.

Multivariate analysis of complex dataset is excellent at discerning correlations within the data material and displaying such correlations in understandable two-dimensional graphs. The unsupervised methods, such as principal component analysis (PCA), show inherent correlations in the data material. The supervised models, such as orthogonal projection to latent structures discriminant analysis (OPLS-DA) attempts to define which variables contribute to a difference between specified classes of data. The OPLS-DA utilizes cross validated (CV)-cycles to validate the model and although many samples are omitted in each CV-cycle, strong groupings may persist and cause the estimate of the predictive power to be overestimated. A possible limitation of the supervised model could be low $R^{2}$ and $Q^{2}$ values, particularly if the model was to be used for class prediction. In this present study the OPLS-DA model was employed to discern overall correlations and differences in the data. A highly significant model was developed, but the $\mathrm{R}^{2}$ and $\mathrm{Q}^{2}$ point to a rather large amount of noise in the data. Small sample size is also problematic, but the model is robust enough for exploratory analysis, which was the purpose in this case. Biomarker discovery for diagnostics naturally requires a higher R2 and Q2, but this was not the aim in this study. Instead multivariate analysis (MVA) was employed to describe the overall correlation patterns in the data. Further studies are required to verify the findings in this present study.

\section{Conclusions}

This study shows an increased expression of pro-inflammatory cytokines occuring in the brain tissue surrounding an intracerebral hemorrhage 20-26 hours following surgical evacuation. This may constitute a target for future therapies aiming to reduce potential secondary brain injury. Microdialysis monitoring of 
the cytokine expression in interstitial fluid may also be a method of monitoring future therapies aimed at reducing the secondary injury caused by inflammation following $\mathrm{ICH}$.

\section{Abbreviations}

$\mathrm{ICH}=$ intracerebral haemorrhage; $\mathrm{ICP}=$ intracranial pressure; IFN = interferon; IL = interleukin; IP-10 = interferon-gamma induced protein 10; LPR = lactate pyruvate ratio; $\mathrm{MCP}=$ monocyte chemoattractant protein; $\mathrm{MD}=$ microdialysis; $\mathrm{MIP}=$ macrophage inflammatory protein; $\mathrm{PHZ}=$ perihemorrhagic zone; $\mathrm{SNX}$ = seemingly normal cortex; TNF = tumor necrosis factor; VEGF-A = vascular endothelial growth factor $A$; VIP = variable of importance in projection .

\section{Declarations}

\section{Ethics approval and consent to participate}

The regional ethical committee in Linköping, Sweden approved the study protocol (decision number 2014/236-31). The study was carried out in accordance with relevant guidelines and regulations, including the World Medical Association (WMA) Declaration of Helsinki. Since the included ICH patients could not themselves consent to the study, a written informed consent was obtained from the patient's closest relative.

\section{Consent for publication}

Not applicable.

\section{Availability of data and materials}

The datasets used and analysed during the current study are available from the corresponding author on reasonable request.

\section{Competing interests}

The authors declare that they have no competing interests.

\section{Funding}

This study was supported by local hospital grants from Region Östergötland, and by grants from the Swedish state under the agreement between the Swedish government and the county councils, the ALFagreement (Lio-925101).

\section{Author contributions}

LT and NM devised the study. AG and KR analysed the inflammatory mediators. LT and NM drafted the manuscript and subsequently all authors edited and revised the manuscript. 
Acknowledgements

The authors wish to thank the patients who were included in the study, and their relatives. The authors also thank Dr Peter Siesjö for valuable opinions on the manuscript.

\section{References}

1. Feigin VL, Norrving B, Mensah GA. Global Burden of Stroke. Circulation research. 2017;120(3):43948.

2. Hemphill JC 3rd, Greenberg SM, Anderson CS, Becker K, Bendok BR, Cushman M, et al. Guidelines for the Management of Spontaneous Intracerebral Hemorrhage: A Guideline for Healthcare Professionals From the American Heart Association/American Stroke Association. Stroke. 2015;46(7):2032-60.

3. Steiner T, Al-Shahi Salman R, Beer R, Christensen H, Cordonnier C, Csiba L, et al. European Stroke Organisation (ESO) guidelines for the management of spontaneous intracerebral hemorrhage. International journal of stroke: official journal of the International Stroke Society. 2014;9(7):840-55.

4. Mendelow AD, Gregson BA, Rowan EN, Murray GD, Gholkar A, Mitchell PM. Early surgery versus initial conservative treatment in patients with spontaneous supratentorial lobar intracerebral haematomas (STICH II): a randomised trial. Lancet. 2013;382(9890):397-408.

5. Prasad K, Mendelow AD, Gregson B. Surgery for primary supratentorial intracerebral haemorrhage. The Cochrane database of systematic reviews. 2008(4):Cd000200.

6. Mendelow AD, Gregson BA, Fernandes HM, Murray GD, Teasdale GM, Hope DT, et al. Early surgery versus initial conservative treatment in patients with spontaneous supratentorial intracerebral haematomas in the International Surgical Trial in Intracerebral Haemorrhage (STICH): a randomised trial. Lancet. 2005;365(9457):387-97.

7. Qureshi Al, Palesch YY, Martin R, Novitzke J, Cruz-Flores S, Ehtisham A, et al. Effect of systolic blood pressure reduction on hematoma expansion, perihematomal edema, and 3-month outcome among patients with intracerebral hemorrhage: results from the antihypertensive treatment of acute cerebral hemorrhage study. Arch Neurol. 2010;67(5):570-6.

8. Qureshi Al, Hanel RA, Kirmani JF, Yahia AM, Hopkins LN. Cerebral blood flow changes associated with intracerebral hemorrhage. Neurosurg Clin North Am. 2002;13(3):355-70.

9. Qureshi Al, Wilson DA, Hanley DF, Traystman RJ. No evidence for an ischemic penumbra in massive experimental intracerebral hemorrhage. Neurology. 1999;52(2):266-72.

10. Kim-Han JS, Kopp SJ, Dugan LL, Diringer MN. Perihematomal mitochondrial dysfunction after intracerebral hemorrhage. Stroke. 2006;37(10):2457-62.

11. Nilsson OG, Polito A, Saveland H, Ungerstedt U, Nordstrom CH. Are primary supratentorial intracerebral hemorrhages surrounded by a biochemical penumbra? A microdialysis study. Neurosurgery. 2006;59(3):521-8. discussion - 8 . 
12. Zhang Z, Zhang Z, Lu H, Yang Q, Wu H, Wang J. Microglial Polarization and Inflammatory Mediators After Intracerebral Hemorrhage. Mol Neurobiol. 2017;54(3):1874-86.

13. Gao Z, Wang J, Thiex R, Rogove AD, Heppner FL, Tsirka SE. Microglial activation and intracerebral hemorrhage. Acta neurochirurgica Supplement. 2008;105:51-3.

14. Kettenmann H, Hanisch UK, Noda M, Verkhratsky A. Physiology of microglia. Physiol Rev. 2011;91(2):461-553.

15. Taylor RA, Chang CF, Goods BA, Hammond MD, Mac Grory B, Ai Y, et al. TGF- $\beta 1$ modulates microglial phenotype and promotes recovery after intracerebral hemorrhage. J Clin Investig. 2017;127(1):28092.

16. Qureshi Al, Mendelow AD, Hanley DF. Intracerebral haemorrhage. Lancet. 2009;373(9675):1632-44.

17. Sansing LH, Harris TH, Welsh FA, Kasner SE, Hunter CA, Kariko K. Toll-like receptor 4 contributes to poor outcome after intracerebral hemorrhage. Ann Neurol. 2011;70(4):646-56.

18. Aronowski J, Hall CE. New horizons for primary intracerebral hemorrhage treatment: experience from preclinical studies. Neurological research. 2005;27(3):268-79.

19. Lan X, Han X, Liu X, Wang J. Inflammatory responses after intracerebral hemorrhage: From cellular function to therapeutic targets. Journal of cerebral blood flow and metabolism: official journal of the International Society of Cerebral Blood Flow and Metabolism. 2018:271678x18805675.

20. Wang J, Dore S. Inflammation after intracerebral hemorrhage. Journal of cerebral blood flow metabolism: official journal of the International Society of Cerebral Blood Flow Metabolism. 2007;27(5):894-908.

21. Yenari MA, Xu L, Tang XN, Qiao Y, Giffard RG. Microglia potentiate damage to blood-brain barrier constituents: improvement by minocycline in vivo and in vitro. Stroke. 2006;37(4):1087-93.

22. Zhao F, Hua Y, He Y, Keep RF, Xi G. Minocycline-induced attenuation of iron overload and brain injury after experimental intracerebral hemorrhage. Stroke. 2011;42(12):3587-93.

23. Zhou Y, Wang Y, Wang J, Anne Stetler R, Yang QW. Inflammation in intracerebral hemorrhage: from mechanisms to clinical translation. Progress in neurobiology. 2014;115:25-44.

24. Beez T, Steiger HJ, Etminan N. Pharmacological targeting of secondary brain damage following ischemic or hemorrhagic stroke, traumatic brain injury, and bacterial meningitis - a systematic review and meta-analysis. BMC Neurol. 2017;17(1):209.

25. Poungvarin N, Bhoopat W, Viriyavejakul A, Rodprasert P, Buranasiri P, Sukondhabhant S, et al. Effects of dexamethasone in primary supratentorial intracerebral hemorrhage. $\mathrm{N}$ Engl $\mathrm{J}$ Med. 1987;316(20):1229-33.

26. Hillered L, Persson L, Nilsson P, Ronne-Engstrom E, Enblad P. Continuous monitoring of cerebral metabolism in traumatic brain injury: a focus on cerebral microdialysis. Curr Opin Crit Care. 2006;12(2):112-8.

27. Ungerstedt U. Microdialysis-principles and applications for studies in animals and man. Journal of internal medicine. 1991;230(4):365-73. 
28. Diedler J, Karpel-Massler G, Sykora M, Poli S, Sakowitz OW, Veltkamp R, et al. Autoregulation and brain metabolism in the perihematomal region of spontaneous intracerebral hemorrhage: an observational pilot study. Journal of the neurological sciences. 2010;295(1-2):16-22.

29. Tobieson L, Rossitti S, Zsigmond P, Hillman J, Marklund N. Persistent Metabolic Disturbance in the Perihemorrhagic Zone Despite a Normalized Cerebral Blood Flow Following Surgery for Intracerebral Hemorrhage. Neurosurgery. 2018. DOI 10.1093/neuros/nyy179.

30. Winter CD, lannotti F, Pringle AK, Trikkas C, Clough GF, Church MK. A microdialysis method for the recovery of IL-1 beta, IL-6 and nerve growth factor from human brain in vivo. J Neurosci Methods. 2002;119(1):45-50.

31. Hillman J, Aneman O, Anderson C, Sjogren F, Saberg C, Mellergard P. A microdialysis technique for routine measurement of macromolecules in the injured human brain. Neurosurgery. 2005;56(6):12648; discussion 8-70.

32. Clausen F, Marklund N, Hillered L. Acute Inflammatory Biomarker Responses to Diffuse Traumatic Brain Injury in the Rat Monitored by a Novel Microdialysis Technique. J Neurotrauma. 2019;36(2):201-11.

33. Mellergard P, Aneman O, Sjogren F, Saberg C, Hillman J. Differences in Cerebral Extracellular Response of Interleukin-1 beta, Interleukin-6, and Interleukin-10 After Subarachnoid Hemorrhage or Severe Head Trauma in Humans. Neurosurgery. 2011;68(1):12-9.

34. Mellergard P, Sjogren F, Hillman J. Release of VEGF and FGF in the extracellular space following severe subarachnoidal haemorrhage or traumatic head injury in humans. Br J Neurosurg. 2010;24(3):261-7.

35. Hillman J, Aneman O, Persson M, Andersson C, Dabrosin C, Mellergard P. Variations in the response of interleukins in neurosurgical intensive care patients monitored using intracerebral microdialysis. Journal of neurosurgery. 2007;106(5):820-5.

36. Helmy A, Carpenter KL, Menon DK, Pickard JD, Hutchinson PJ. The cytokine response to human traumatic brain injury: temporal profiles and evidence for cerebral parenchymal production. Journal of cerebral blood flow metabolism: official journal of the International Society of Cerebral Blood Flow Metabolism. 2011;31(2):658-70.

37. Helmy A, Carpenter KL, Skepper JN, Kirkpatrick PJ, Pickard JD, Hutchinson PJ. Microdialysis of cytokines: methodological considerations, scanning electron microscopy, and determination of relative recovery. J Neurotrauma. 2009;26(4):549-61.

38. Dyhrfort P, Shen Q, Clausen F, Thulin M, Enblad P, Kamali-Moghaddam M, et al. Monitoring of Protein Biomarkers of Inflammation in Human Traumatic Brain Injury Using Microdialysis and Proximity Extension Assay Technology in Neurointensive Care. J Neurotrauma. 2019;36(20):2872-85.

39. Dziedzic T, Bartus S, Klimkowicz A, Motyl M, Slowik A, Szczudlik A. Intracerebral hemorrhage triggers interleukin-6 and interleukin-10 release in blood. Stroke. 2002;33(9):2334-5.

40. Kim JS, Yoon SS, Kim YH, Ryu JS. Serial measurement of interleukin-6, transforming growth factorbeta, and S-100 protein in patients with acute stroke. Stroke. 1996;27(9):1553-7. 
41. Castillo J, Davalos A, Alvarez-Sabin J, Pumar JM, Leira R, Silva Y, et al. Molecular signatures of brain injury after intracerebral hemorrhage. Neurology. 2002;58(4):624-9.

42. Silva Y, Leira R, Tejada J, Lainez JM, Castillo J, Davalos A. Molecular signatures of vascular injury are associated with early growth of intracerebral hemorrhage. Stroke. 2005;36(1):86-91.

43. Engstrom M, Polito A, Reinstrup P, Romner B, Ryding E, Ungerstedt U, et al. Intracerebral microdialysis in severe brain trauma: the importance of catheter location. Journal of neurosurgery. 2005;102(3):460-9.

44. Hutchinson $P, O^{\prime} P h e l a n ~ K$. International multidisciplinary consensus conference on multimodality monitoring: cerebral metabolism. Neurocritical care. 2014;21 Suppl 2:S148-58.

45. van Swieten JC, Koudstaal PJ, Visser MC, Schouten HJ, van Gijn J. Interobserver agreement for the assessment of handicap in stroke patients. Stroke. 1988;19(5):604-7.

46. Quinn TJ, Dawson J, Walters MR, Lees KR. Reliability of the modified Rankin Scale: a systematic review. Stroke. 2009;40(10):3393-5.

47. Hutchinson PJ, Jalloh I, Helmy A, Carpenter KL, Rostami E, Bellander BM, et al. Consensus statement from the 2014 International Microdialysis Forum. Intensive care medicine. 2015;41(9):1517-28.

48. Hillman J, Milos P, Yu ZQ, Sjogren F, Anderson C, Mellergard P. Intracerebral microdialysis in neurosurgical intensive care patients utilising catheters with different molecular cut-off ( 20 and 100 kD). Acta Neurochir. 2006;148(3):319-24. discussion 24.

49. Hutchinson PJ, O'Connell MT, Nortje J, Smith P, Al-Rawi PG, Gupta AK, et al. Cerebral microdialysis methodology-evaluation of $20 \mathrm{kDa}$ and $100 \mathrm{kDa}$ catheters. Physiological measurement. 2005;26(4):423-8.

50. Wheelock AM, Wheelock CE. Trials and tribulations of 'omics data analysis: assessing quality of SIMCA-based multivariate models using examples from pulmonary medicine. Molecular bioSystems. 2013;9(11):2589-96.

51. Mayne M, Ni W, Yan HJ, Xue M, Johnston JB, Del Bigio MR, et al. Antisense oligodeoxynucleotide inhibition of tumor necrosis factor-alpha expression is neuroprotective after intracerebral hemorrhage. Stroke. 2001;32(1):240-8.

52. Xi G, Hua Y, Bhasin RR, Ennis SR, Keep RF, Hoff JT. Mechanisms of edema formation after intracerebral hemorrhage: effects of extravasated red blood cells on blood flow and blood-brain barrier integrity. Stroke. 2001;32(12):2932-8.

53. Lu A, Tang Y, Ran R, Ardizzone TL, Wagner KR, Sharp FR. Brain genomics of intracerebral hemorrhage. Journal of cerebral blood flow metabolism: official journal of the International Society of Cerebral Blood Flow Metabolism. 2006;26(2):230-52.

54. Wagner KR, Beiler S, Beiler C, Kirkman J, Casey K, Robinson T, et al. Delayed profound local brain hypothermia markedly reduces interleukin-1beta gene expression and vasogenic edema development in a porcine model of intracerebral hemorrhage. Acta neurochirurgica Supplement. 2006;96:177-82.

55. Engelhardt S, Patkar S, Ogunshola 00. Cell-specific blood-brain barrier regulation in health and disease: a focus on hypoxia. Br J Pharmacol. 2014;171(5):1210-30. 
56. Zhang ZG, Zhang L, Jiang Q, Zhang R, Davies K, Powers C, et al. VEGF enhances angiogenesis and promotes blood-brain barrier leakage in the ischemic brain. J Clin Investig. 2000;106(7):829-38.

57. Helmy A, Guilfoyle MR, Carpenter KL, Pickard JD, Menon DK, Hutchinson PJ. Recombinant human interleukin-1 receptor antagonist promotes M1 microglia biased cytokines and chemokines following human traumatic brain injury. Journal of cerebral blood flow metabolism: official journal of the International Society of Cerebral Blood Flow Metabolism. 2016;36(8):1434-48.

58. Lan X, Han X, Li Q, Yang QW, Wang J. Modulators of microglial activation and polarization after intracerebral haemorrhage. Nature reviews Neurology. 2017;13(7):420-33.

59. Liu X, Liu J, Zhao S, Zhang H, Cai W, Cai M, et al. Interleukin-4 Is Essential for Microglia/Macrophage M2 Polarization and Long-Term Recovery After Cerebral Ischemia. Stroke. 2016;47(2):498-504.

60. Marín-Teva JL, Cuadros MA, Martín-Oliva D, Navascués J. Microglia and neuronal cell death. Neuron Glia Biol. 2011;7(1):25-40.

61. Mracsko E, Veltkamp R. Neuroinflammation after intracerebral hemorrhage. Frontiers in cellular neuroscience. 2014;8:388.

62. Wang J. Preclinical and clinical research on inflammation after intracerebral hemorrhage. Progress in neurobiology. 2010;92(4):463-77.

63. Zhao X, Grotta J, Gonzales N, Aronowski J. Hematoma resolution as a therapeutic target: the role of microglia/macrophages. Stroke. 2009;40(3 Suppl):92-4.

64. Gordon S. Alternative activation of macrophages. Nat Rev Immunol. 2003;3(1):23-35.

65. Pelegrin P, Surprenant A. Dynamics of macrophage polarization reveal new mechanism to inhibit IL1 beta release through pyrophosphates. Embo j. 2009;28(14):2114-27.

66. Lopez-Castejón G, Baroja-Mazo A, Pelegrín P. Novel macrophage polarization model: from gene expression to identification of new anti-inflammatory molecules. Cell Mol Life Sci. 2011;68(18):3095-107.

67. Cavaillon JM. Pro- versus anti-inflammatory cytokines: myth or reality. Cellular and molecular biology (Noisy-le-Grand. France). 2001;47(4):695-702.

68. Commins SP, Borish L, Steinke JW. Immunologic messenger molecules: cytokines, interferons, and chemokines. J Allergy Clin Immunol. 2010;125(2 Suppl 2):53-72.

69. Hutchinson PJ, O'Connell MT, Rothwell NJ, Hopkins SJ, Nortje J, Carpenter KL, et al. Inflammation in human brain injury: intracerebral concentrations of IL-1 alpha, IL-1beta, and their endogenous inhibitor IL-1ra. J Neurotrauma. 2007;24(10):1545-57.

70. Shiozaki T, Hayakata T, Tasaki O, Hosotubo H, Fuijita K, Mouri T, et al. Cerebrospinal fluid concentrations of anti-inflammatory mediators in early-phase severe traumatic brain injury. Shock. 2005;23(5):406-10.

71. Ungerstedt U, Rostami E. Microdialysis in neurointensive care. Curr Pharm Design. 2004;10(18):2145-52. 
72. Mellergard P, Aneman O, Sjogren F, Pettersson P, Hillman J. Changes in extracellular concentrations of some cytokines, chemokines, and neurotrophic factors after insertion of intracerebral microdialysis catheters in neurosurgical patients. Neurosurgery. 2008;62(1):151-7. discussion 7-8.

73. Fahlstrom A, Tobieson L, Redebrandt HN, Zeberg H, Bartek J Jr, Bartley A, et al. Differences in neurosurgical treatment of intracerebral haemorrhage: a nation-wide observational study of 578 consecutive patients. Acta neurochirurgica. 2019.

74. Mayne M, Fotheringham J, Yan HJ, Power C, Del Bigio MR, Peeling J, et al. Adenosine A2A receptor activation reduces proinflammatory events and decreases cell death following intracerebral hemorrhage. Ann Neurol. 2001;49(6):727-35.

75. Masada T, Hua Y, Xi G, Yang GY, Hoff JT, Keep RF. Attenuation of intracerebral hemorrhage and thrombin-induced brain edema by overexpression of interleukin-1 receptor antagonist. Journal of neurosurgery. 2001;95(4):680-6.

76. Masada T, Hua Y, Xi G, Yang GY, Hoff JT, Keep RF, et al. Overexpression of interleukin-1 receptor antagonist reduces brain edema induced by intracerebral hemorrhage and thrombin. Acta neurochirurgica Supplement. 2003;86:463-7.

\section{Tables}

Table 1. Patient characteristics.

\begin{tabular}{|c|c|c|c|c|c|c|c|c|c|}
\hline $\begin{array}{l}\text { Patient } \\
\text { No }\end{array}$ & Sex & $\begin{array}{l}\text { Age } \\
\text { (years) }\end{array}$ & $\begin{array}{l}\text { Time to } \\
\text { surgery } \\
\text { (hours) }\end{array}$ & $\begin{array}{l}\text { Sampling } \\
\text { time } \\
\text { (hours) }\end{array}$ & $\begin{array}{l}\text { Dist- } \\
\text { PHZ } \\
(\mathrm{mm})\end{array}$ & $\begin{array}{l}\text { Dist- } \\
\text { SNX } \\
(\mathrm{mm})\end{array}$ & $\begin{array}{l}\text { Volume } \\
\text { (mL) }\end{array}$ & $\begin{array}{l}\text { Side } \\
\text { of } \\
\text { ICH }\end{array}$ & $\begin{array}{l}\text { ICH } \\
\text { Location }\end{array}$ \\
\hline 1 & $M$ & 51 & 10 & 112 & 0 & 27 & 89 & L & BG \\
\hline 2 & $M$ & 71 & 14 & 74 & 6 & 38 & 93 & $\mathrm{R}$ & L \\
\hline 3 & $M$ & 64 & 82 & 148 & 6 & 20 & 43 & $\mathrm{R}$ & BG \\
\hline 4 & $F$ & 70 & 6 & 58 & 4 & 22 & 89 & L & L \\
\hline 5 & $M$ & 68 & 6 & 130 & 2 & 34 & 114 & $\mathrm{R}$ & $B G$ \\
\hline 6 & $M$ & 64 & 6 & 86 & 8 & 30 & 45 & L & $B G$ \\
\hline 7 & $M$ & 63 & 58 & 146 & 9 & 11 & 44 & L & $B G$ \\
\hline 8 & $M$ & 69 & 20 & 50 & 6 & 19 & 78 & $\mathrm{R}$ & L \\
\hline 9 & $F$ & 64 & 12 & 90 & 0 & 27 & 33 & L & $B G$ \\
\hline 10 & $M$ & 57 & 64 & 56 & 18 & 19 & 76 & L & $\mathrm{BG}$ \\
\hline
\end{tabular}


Table 2. Variables contributing strongly to OPLS-DA model discriminating between perihemorrhagic zone (PHZ) and seemingly normal cortex (SNX).

\begin{tabular}{|lll|}
\hline Variable & $\mathbf{p}$ (corr) & VIP \\
\hline Lactate & -0.77687 & 1.82257 \\
\hline IL-13 & -0.70948 & 1.66652 \\
\hline IL-8 & -0.6973 & 1.63755 \\
\hline Glutamate & -0.68288 & 1.61133 \\
\hline TNF-a & -0.66947 & 1.57238 \\
\hline IL-2 & -0.66011 & 1.55044 \\
\hline LPR & -0.65954 & 1.52502 \\
\hline VEGF-A & -0.63103 & 1.48236 \\
\hline IL-4 & -0.58531 & 1.37511 \\
\hline IL-1 $\beta$ & -0.55034 & 1.29242 \\
\hline IL-6 & -0.54935 & 1.29079 \\
\hline IFN-Y & -0.50683 & 1.19044 \\
\hline Pyruvate & -0.46304 & 1.07022 \\
\hline $\begin{array}{l}\text { VIP }>\text { 1 and absolute } p(\text { corr) }>0.4 \text { are used to determine which variables are most important for the } \\
\text { model,(50) meaning these variables contribute most to model group separation. } \\
\text { Abbreviations: VIP = variable of importance in projection; IL = interleukin; TNF = tumor necrosis factor; } \\
\text { VEGF-A = vascular endothelial growth factor A; LPR = lactate pyruvate ratio; IFN = interferon. }\end{array}$ \\
\hline
\end{tabular}

\section{Figures}



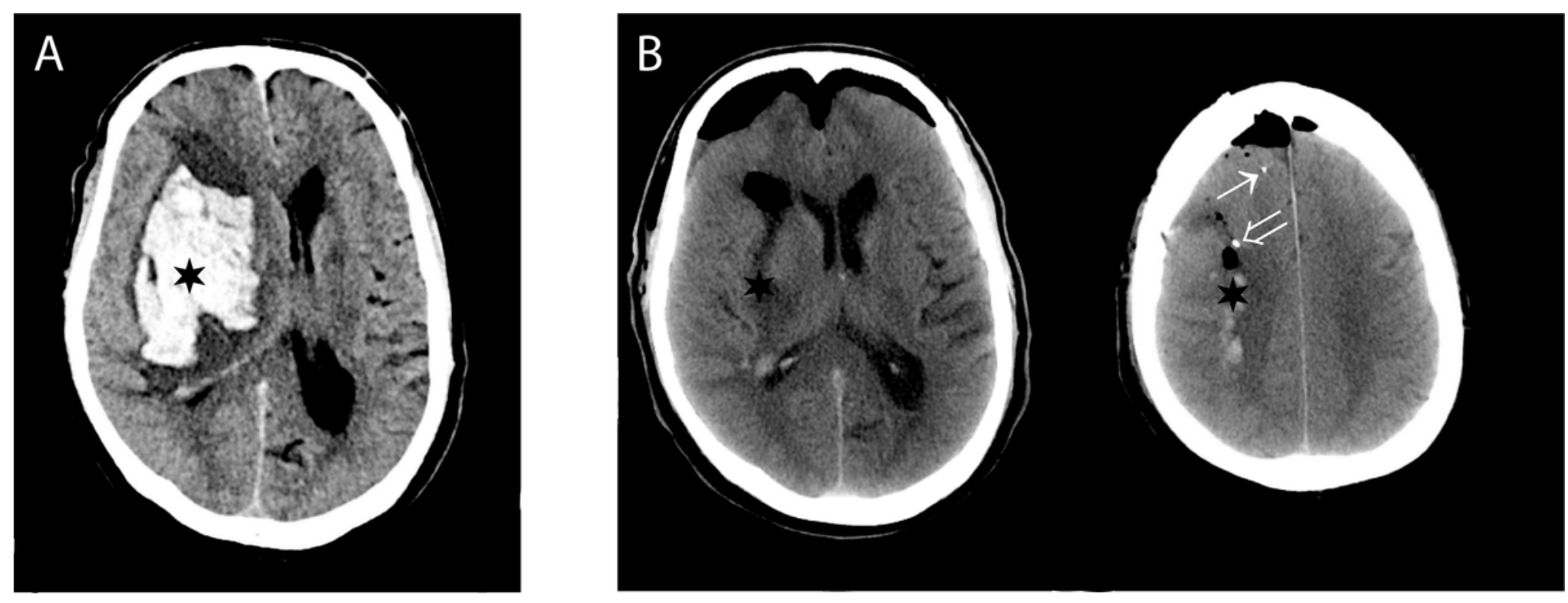

Figure 1

(A) Preoperative CT-scan of a 68 year-old male presenting with an intracerebral hemorrhage (ICH; black star) in the right basal ganglia. (B) Following surgical evacuation of the $\mathrm{ICH}$ a post-operative CT-scan shows the hematoma cavity (black star) and the tip of the microdialysis (MD) catheters placed in the perihemorragic zone (PHZ; open arrow) and seemingly normal cortex (SNX; closed arrow), respectively. 

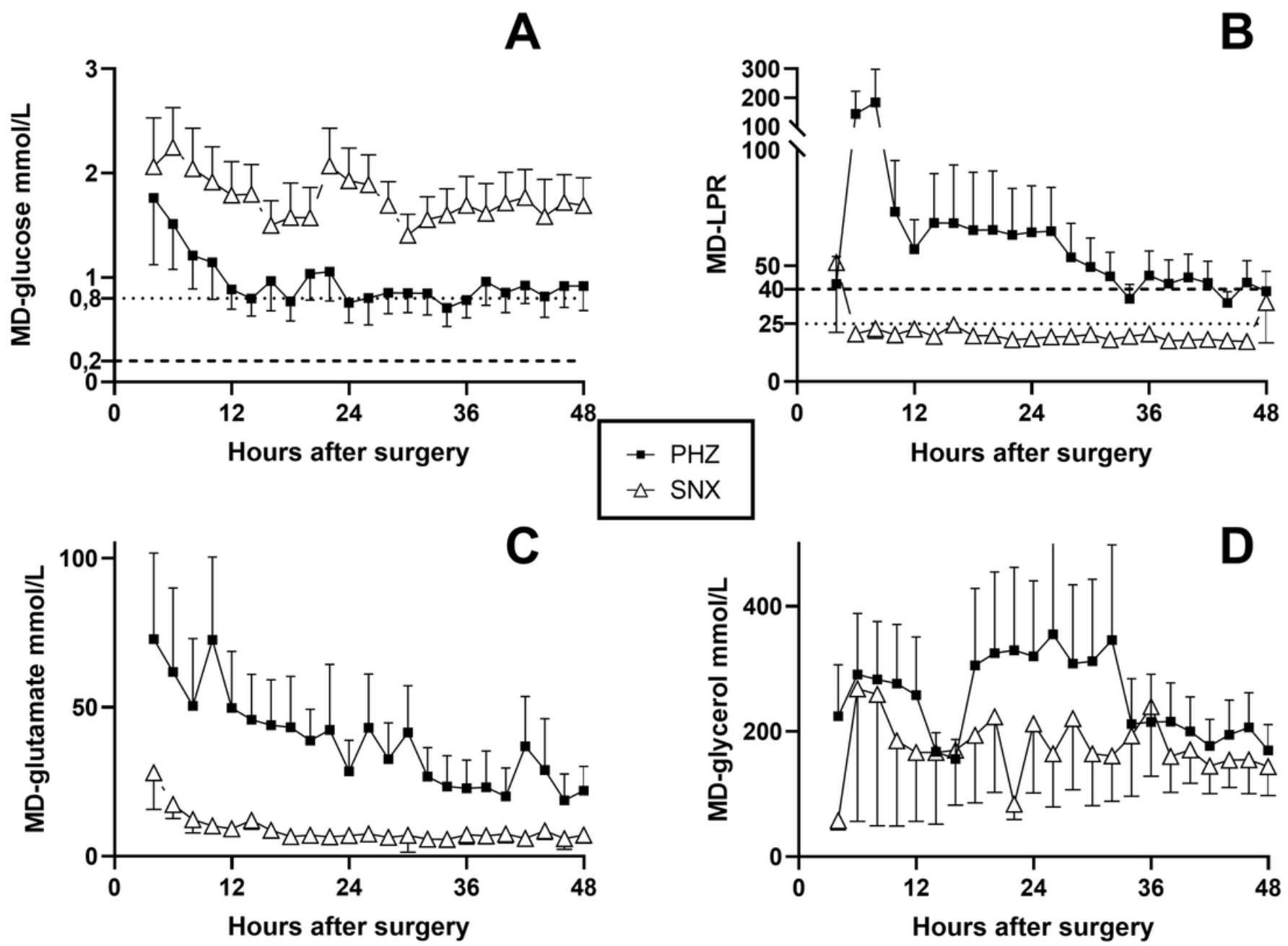

Figure 2

Microdialysis (MD)-glucose (A) levels were significantly lower in the perihemorrhagic zone (PHZ) when compared to those in seemingly normal cortex (SNX) $(p<0.05)$ although consistently above critical $(0.2$ $\mathrm{mmol} / \mathrm{L})$ and warning levels $(0.8 \mathrm{mmol} / \mathrm{L})$ in both locations.(47) The LPR (B), however, was pathologically elevated in the $\mathrm{PHZ}$ indicating a metabolic crisis in the brain tissue. In contrast, the SNX LPR normalized within the first hours after surgery and thereafter remained within the normal range (B). MD-glutamate levels decreased with time in the PHZ but was significantly higher than in the SNX ( $\mathrm{C} ; \mathrm{p}<$ 0.05 ) during the initial 48 hours. In addition, MD-glycerol levels were persistently higher in the PHZ when compared to the SNX ( $; ; p<0.05)$. Abbreviations: LPR = lactate pyruvate ratio. $D$ ata are presented as mean and standard error of the mean (SEM) for clarity. 


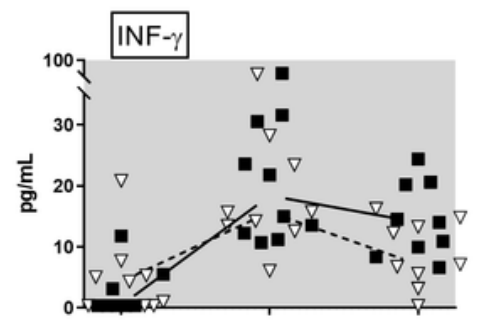

$\mathrm{PHZ} \rightarrow$ Median $\mathrm{PHZ}$

$\nabla$ SNX $-\nabla \cdot$ Median SNX
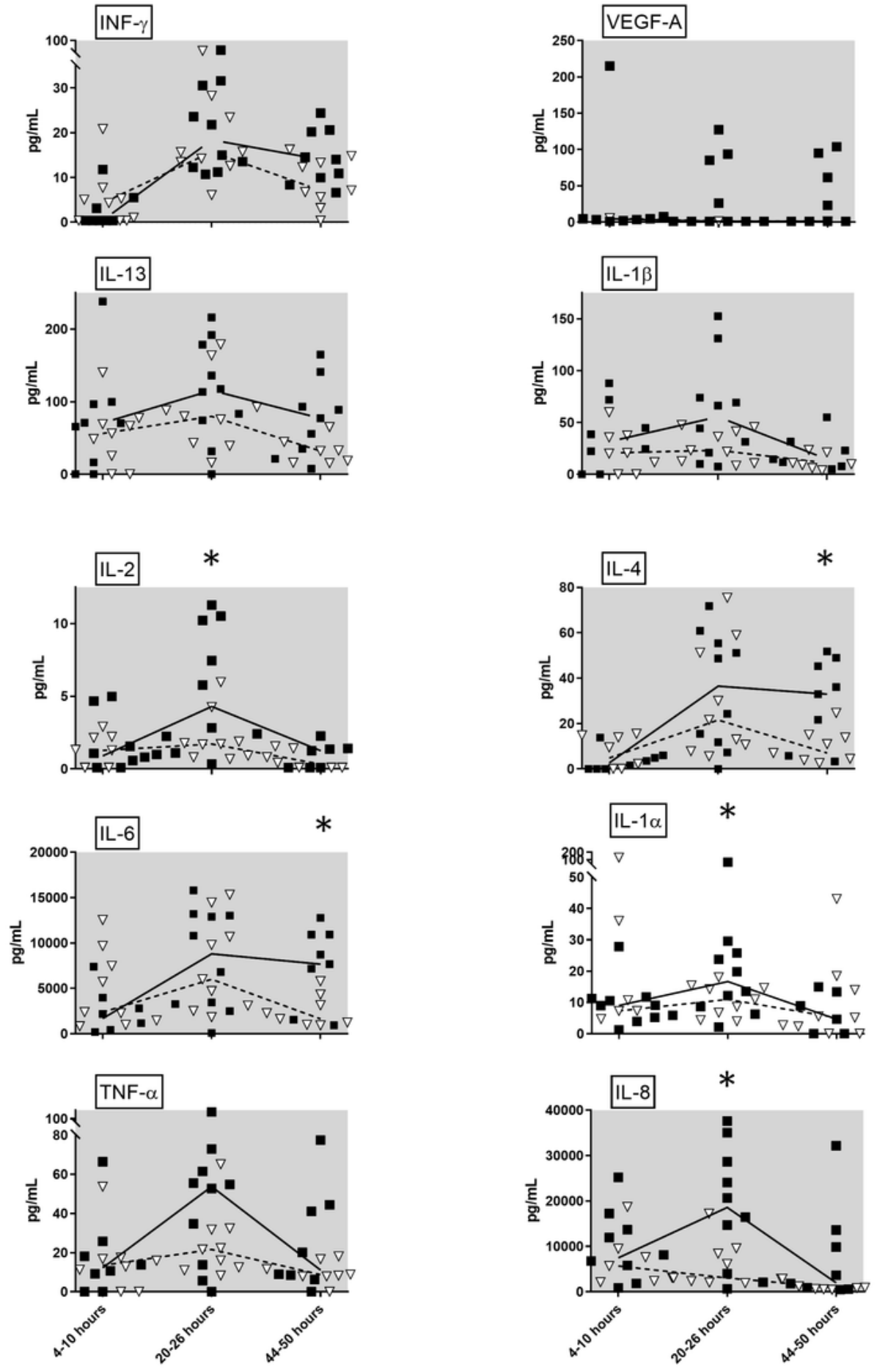

\section{Figure 3}

Univariate analysis of cytokines (median and individual values; ${ }^{*}=p<0.05$ ). There was a significantly higher expression of IL-2 and IL-8 levels in the perihemorrhagic zone (PHZ) when compared to those in the seemingly normal cortex (SNX) at 20-26 hours post-surgery. At 44-50 hours post-surgery, there was a significantly higher expression of IL-4, considered an anti-inflammatory cytokine, and IL-6, considered a pro-inflammatory cytokine, in the PHZ when compared to the SNX. Several of the cytokines contributing 
to the group separation between PHZ and SNX in the supervised OPLS-DA model (highlighted with grey background in this figure) did not reach statistically significant differences when explored using univariate statistical methods. In contrast, IL-1 a was significantly higher in PHZ at 20-26 hours after surgery ( $p<0.05$; Wilcoxon signed rank) in univariate analysis, but did not contribute to the multivariate model.

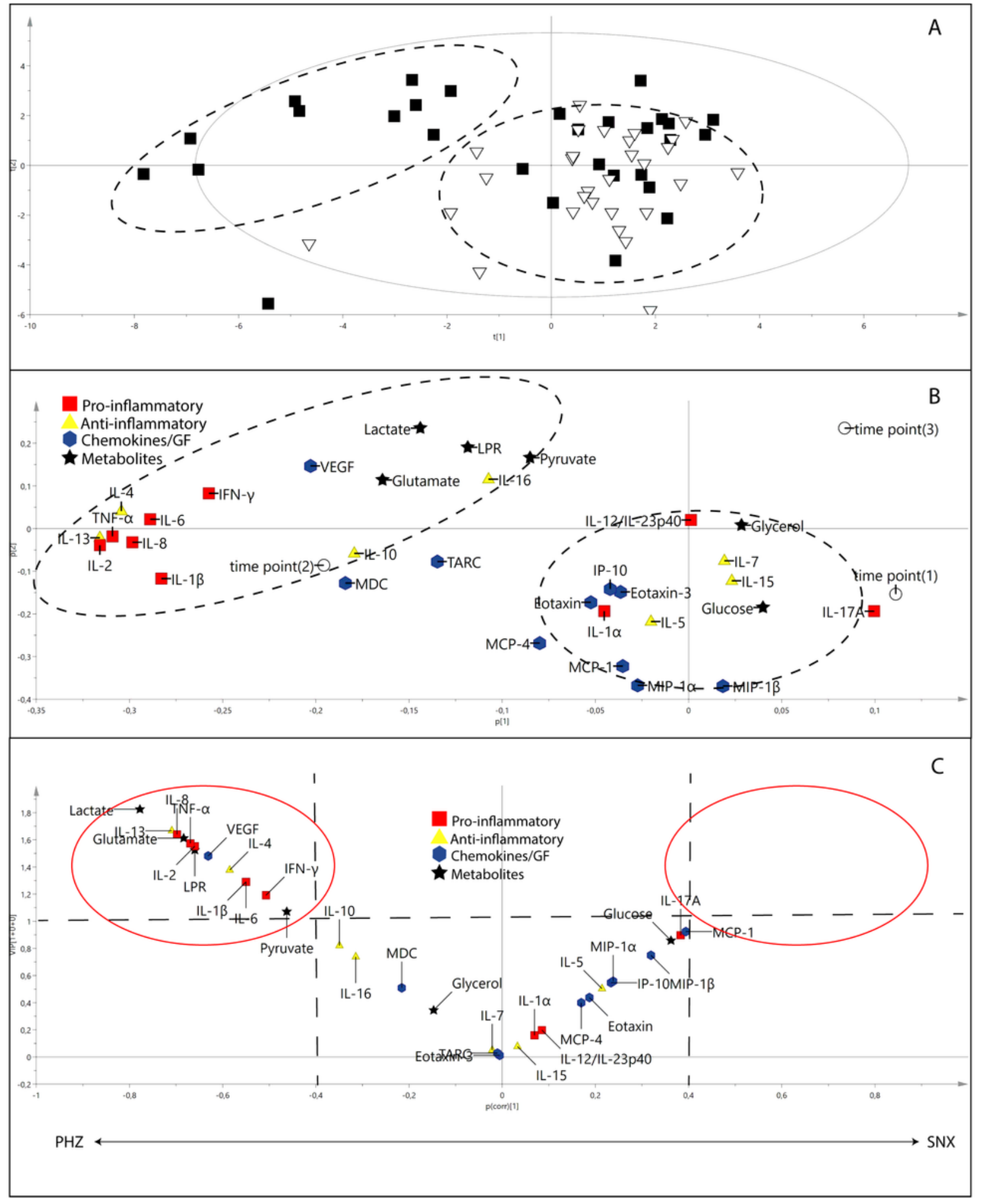

Figure 4 
Multivariate data analysis showed group separation between perihemorrhagic zone ( $\mathrm{PHZ}$ ) and seemingly normal cortex (SNX). (A) Principal component analysis (PCA) score scatter plot of cytokine and metabolite data showing separation between PHZ (black squares) and SNX (white inverted triangles). $\mathrm{PHZ}$ data points gravitate towards the upper and left quadrant, whereas SNX data points predominantly cluster towards the lower right quadrant; highlighted with dashed black circles. (B) Corresponding PCA loading scatter plot show clustering of pro-inflammatory cytokines (red squares) and metabolites associated with a deranged metabolic state of the tissue (black stars) to the predominant location of PHZ data (black dashed circle) in figure 4a. In contrast, MD-glucose and MD-glycerol levels cluster with SNX data points, as do chemokines (blue hexagons) and anti-inflammatory cytokines (yellow triangles). Time point 2 (20-26 hours post-surgery; unfilled circle) correlates with higher pro-inflammatory cytokine expression compared to time point 1 (4-10 hours post-surgery) and 3 (44-50 hours post-surgery) respectively, apart from IL-17A which is distinctly correlated with time point 1. (C) Volcano plot for the OPLS-DA model with one latent variable. Variables associated with $\mathrm{PHZ}$ display to the left on the $\mathrm{x}$-axis and those associated with SNX to the right. Variables which contribute strongest to group discrimination (i.e. variables with a $\mid p($ corr $) \mid>0.4$ and $V I P>1$ ) place within the red circles. Lactate, pyruvate, LPR and glutamate (black); pro-inflammatory cytokines IL-8, TNF-a, IL-2, IL-1 $\beta$, IL-6 and IFN- $($ (red); antiinflammatory cytokines IL-13 and IL-4 (yellow); and growth factor VEGF-A (blue) where all higher in the $\mathrm{PHZ}$ compared to SNX, illustrated by fact that all significant variables place in the left of the two red circles (also listed in Table 2). The majority of the anti-inflammatory cytokines and chemokines did not contribute significantly to the model, thus suggesting that their concentrations were similar in the PHZ and the SNX.

\section{Supplementary Files}

This is a list of supplementary files associated with this preprint. Click to download.

- supplementalfigure1.tif

- supplementalfigure2.tif 